\title{
Multiresolution-based reconstruction for compressive spectral video sensing using a spectral multiplexing sensor
}

\section{Reconstrucción basada en multirresolución para la adquisición de vídeo espectral compresivo usando un sensor de multiplexación espectral}

\author{
Kareth León ${ }^{1}$, Laura Galvis ${ }^{2}$, Henry Arguello ${ }^{3}$ \\ ${ }^{1}$ Department of Computer Science, Universidad Industrial de Santander, Bucaramanga, Colombia. \\ Email: kareth.leon@correo.uis.edu.co \\ ${ }^{2}$ Department of Electrical and Computer Engineering, University of Delaware, Newark, United States. Email: 1galvis@udel.edu \\ ${ }^{3}$ Department of Computer Science, Universidad Industrial de Santander, Bucaramanga, Colombia. \\ Email: henarfu@uis.edu.co
}

RECEIVED: February 20, 2017. ACCEPTED: May 27, 2017. FinAL VERSION: October 5, 2017.

\begin{abstract}
Spectral multiplexing sensors based on compressive sensing attempt to break the Nyquist barrier to acquire high spectral resolution scenes. Particularly, the colored coded aperture-based compressive spectral imager extended to video, or video C-CASSI, is a spectral multiplexing sensor that allows capturing spectral dynamic scenes by projecting each spectral frame onto a bidimensional detector using a 3D coded aperture. Afterwards, the compressed signal reconstruction is performed iteratively by finding a sparse solution to an undetermined linear system of equations. Even though the acquired signal can be recovered from much fewer observations by an $\ell_{2}-\ell_{1}$-norm recovery algorithm than using conventional sensors, the reconstruction exhibits diverse challenges originated by the temporal variable or motion. The motion during the reconstruction produces artifacts that damages the entire data. In this work, a multiresolution-based reconstruction method for compressive spectral video sensing is proposed. In this way, it obtains the temporal information from the measurements at a low computational cost. Thereby, the optimization problem to recover the signal is extended by adding temporal information in order to correct the errors originated by the scene motion. Computational experiments performed over four different spectral videos show an improvement up to $4 \mathrm{~dB}$ in terms of peak-signal to noise ratio (PSNR) in the reconstruction quality using the multiresolution approach applied to the spectral video reconstruction with respect to the traditional inverse problem.
\end{abstract}

KEYWORDS: Multiresolution reconstruction; compressive spectral video; optimization.

\section{RESUMEN}

Los sensores de multiplexación espectral basados en muestreo compresivo intentan romper la barrera de Nyquist para adquirir escenas de alta resolución espectral. Particularmente, el sistema de imágenes espectrales de única captura basado en aperturas codificadas de color extendido a vídeo, o video - CCASSI, es un sensor de multiplexación espectral que permite la adquisición de imágenes espectrales dinámicas proyectando cada fotograma espectral sobre un detector bidimensional usando un apertura de codificación 3D. Posteriormente, la reconstrucción de la señal

ISSN Impreso: 1657 - 4583, En Línea: 2145 - 8456

Este artículo puede compartirse bajo la licencia CC BY-ND 4.0 y se referencia usando el siguiente formato: K. León, L. Galvis, H. Arguello, "Multiresolution-based reconstruction for compressive spectral video sensing using a spectral multiplexing sensor," Rev. UIS Ing., vol. 17, no. 1, pp. 209-216, 2018. Doi: https://doi.org/10.18273/revuin.v17n1-2018020 
comprimida se realiza iterativamente encontrando una solución escasa a un sistema lineal de ecuaciones indeterminado. Si bien la señal adquirida puede ser recuperada desde un algoritmo basado en la norma 1_2 - 1_1, con muchas menos observaciones en comparación a los sistemas convencionales, dicha reconstrucción presenta diversos desafíos originados por la variable temporal o el movimiento. El movimiento durante la reconstrucción produce artefactos que dañan la totalidad de los datos. En este trabajo, se propone un método de reconstrucción basado en múltiples resoluciones para la adquisición compresiva de video espectral. De este modo, el problema de optimización para recuperar la señal se amplía añadiendo la información temporal como restricción, con el objetivo de corregir los errores originados por el movimiento de la escena. Los experimentos computacionales realizados en cuatro videos espectrales diferentes muestran una mejora de hasta $4 \mathrm{~dB}$ en términos de relación pico-señal a ruido (PSNR) en la calidad de reconstrucción usando el enfoque multirresolución aplicado a la reconstrucción de video espectral con respecto al problema de optimización que no considera el movimiento.

PALABRAS CLAVE: Reconstrucción basada en multirresolución; video espectral compresivo; optimización.

\section{INTRODUCTION}

Compressive sensing (CS) establishes that a given signal can be recovered from far fewer samples than those required by the Shannon-Nyquist criterion. As a consequence, through the years some sensors to acquire multidimensional signals as spectral images or video from less samples have been developed. Particularly, spectral multiplexing sensors based on CS attempt to break the Nyquist barrier by acquiring 2D projections of a scene in order to obtain a high spectral resolution image. The most remarkable CS sensor for spectral imaging is called the coded aperture snapshot spectral imager (CASSI) which is composed by a few set of elements such as a lenses set, a coded aperture, a dispersive element and a focal plane array (FPA). Particularly, the colored CASSI or C-CASSI is a version of CASSI where the coded aperture is a colored coded aperture (CCA) which leads a richer encoding procedure $[1,2,3]$.

Figure 1 illustrates the set of elements in the C-CASSI. The principal characteristic of a CCA relies on the fact that each CCA pixel can spectrally encode the incoming light letting to pass just a desired set of wavelengths. Hence, the CCA pixels can operate on the spectral axis as frequency-selective filters, i.e. as low pass (L), band pass (B) or high pass (H) optical filters. In other words, each pixel lets to pass certain frequency components of the source pixel and totally rejects all others. Thus, each CCA pixel is one of many possible optical filters whose spectral response can be selected. Figure 1(b) shows an illustration of the above-mentioned filters where each CCA pixel color corresponds to a specific spectral response: Low, High and Band pass filter, respectively. Further, the filters in the CCA can be selected at a randomly form or they can be optimally selected such that the number of projections is minimized while the quality of reconstruction is maximized [1].

The C-CASSI permits the compressive acquisition of a 3D spectral image into a 2D detector. Further, the CCASSI extended to video (video C-CASSI) is a spectral multiplexing sensor that allows capturing spectral dynamic scenes, or spectral video, by projecting each spectral frame onto a bidimensional detector $[4,5,6]$. A spectral video is considered as a four-dimensional signal $f(x, y, \lambda, \tau)$, where $x, y$ denote the spatial pixels, $\lambda$ represents the spectral dimension and $\tau$ denotes the temporal component.

The spectral video has many applications in the industry and the academy, such as surveillance, moving targets recognition, security, and classification, where the discrimination of the features is performed over the different spectral bands instead of use only three channels (RGB) as in traditional approaches $[7,8,9,10]$. Figure 2 shows the sensing process in the video C-CASSI system for a spectral video. Basically, in the sensing process, the incoming light is encoded by the coded aperture $T(x, y, \lambda, \tau)$, and then, the coded light is spectrally dispersed by the dispersive element, usually a prism.

Finally, the encoded and dispersed light is integrated in the FPA.The compressed video is reconstructed iteratively by finding a sparse solution to an undetermined linear system of equations. 


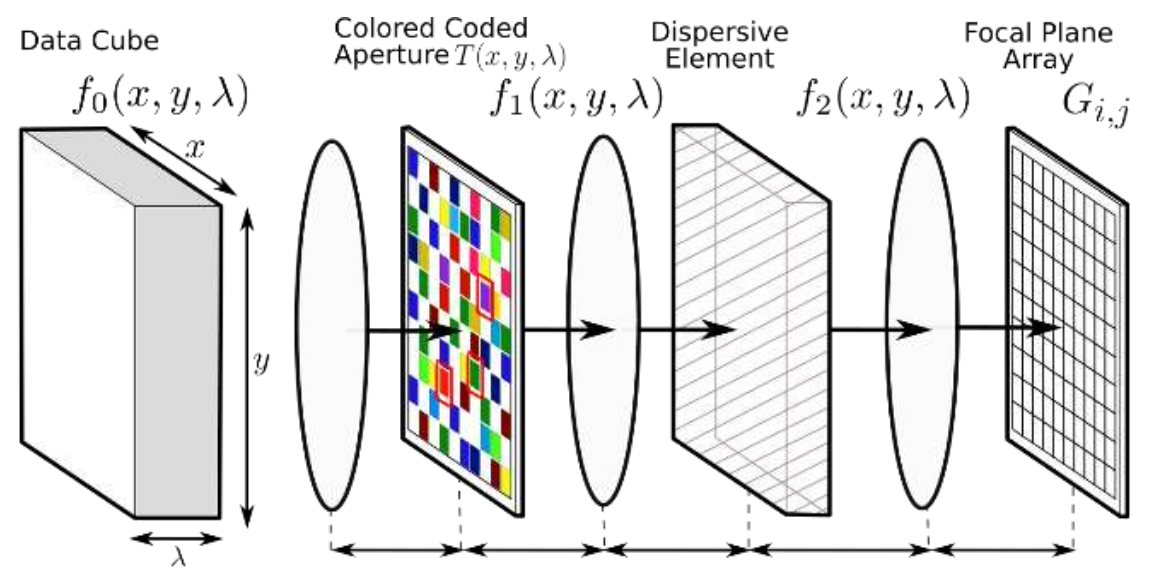

(a) C-CASSI Optical Architecture
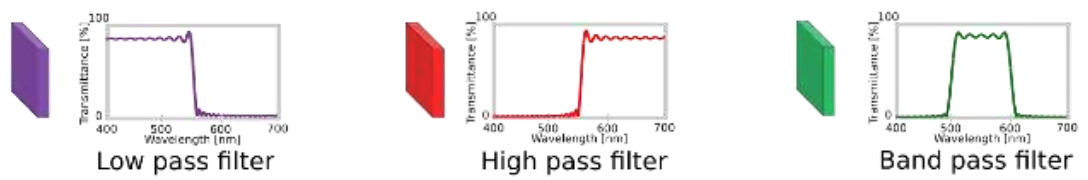

(b) Spectral responses of each optical filter

Figure 1. Optical elements presented in C-CASSI [1]. (a) The binary CA is replaced by a colored coded aperture (CCA). (b) Then, each CCA pixel is one of many possible optical filters whose spectral response can be selected.

However, recovering a compressed video entails diverse challenges originated by the temporal variable. The scene motion during the acquisition yields to motion artifacts, and these artifacts get aliased during the video reconstruction damaging the entire data [11]. As a result, multiresolution approaches have been proposed in order to alleviate the aliasing and enhance the video reconstruction. The idea of interpret the data at multiple resolutions have been called the "chicken-and-egg" problem, which states that reconstructing a high-quality CS video could be obtained adding temporal correlation such as motion compensation, and computing motion compensation requires knowledge of the full video.

Works such as $[12,13]$ propose a preview reconstruction to estimate the motion field in the video such that it can be used to achieve a high-quality reconstruction. However, these approaches have been focused in spatial or temporal multiplexing architectures while the spectral information in the video has been discarded.

This work presents a modification to the compressive spectral video recovery step adding an additional regularization term to correct the errors induced by the motion. Thus, the motion estimated from a low spatial resolution version, or a preview, is imposed as prior information in the optimization problem. This approach aims to correct the artifacts induced by the motion in the reconstruction problem following a multiresolution strategy. Hence, this scheme allows going from a low to a high spatial resolution in the reconstruction, in order to obtain an improvement in the spatial quality of the reconstructed spectral video. In the following sections, it is introduced the discrete model of the CASSI system using colored coded apertures extended to spectral video acquisition. Next, the multiresolution strategy for compressive spectral video reconstruction is developed, and then, a quantitative comparison to measure the performance of the proposed approach is presented.

\section{COMPRESSIVE SPECTRAL VIDEO C-CASSI MODELING}

\subsection{Discrete sampling model for video C-CASSI}

Let $\mathbf{F} \in \mathbb{R}^{N \times N \times L \times D}$ be the discretized-form of a given spectral video, with $N \times N$ spatial pixels, $L$ spectral bands and $D$ spectral frames; then, the sensing process of $\mathbf{F}$ through the video C-CASSI system can be modeled as the linear projection of the vectorized form of the source $\mathbf{f}=\left[\mathbf{f}_{0}^{T}, \mathbf{f}_{1}^{T}, \ldots, \mathbf{f}_{D-1}^{T}\right]^{T}$, where $\mathbf{f} \in \mathbb{R}^{n}$ with $n=N^{2} L D$, onto the matrix $\mathbf{H}$ as

$$
\mathbf{g}=\mathbf{H f} \text {, }
$$

where $\mathbf{g} \in \mathbb{R}^{m}$ represents the vectorized form of the compressive measurements on the detector, with $m=$ $N(N+L-1) D$. For the recovery of the compressive spectral video, CS exploits the fact that many signals can be represented in a sparse form in some representation basis. Formally, the given spectral video signal $\mathbf{f}$ can be expressed as $\mathbf{f}=\boldsymbol{\Psi} \boldsymbol{\theta}$, where $\boldsymbol{\Psi} \in \mathbb{R}^{n \times n}$ is a representation basis such as a Wavelet or Cosine, and $\boldsymbol{\theta}$ denotes the nonzero coefficients of the signal in the given basis $\boldsymbol{\Psi}$. 
Hence, the Eq. (1) can be rewritten as

$\mathbf{g}=\mathbf{H} \Psi \boldsymbol{\theta}$.

\subsection{Inverse problem}

Solving the problem in Eq. (1) requires the inversion of the linear system, however, since the number of the measurements $\mathbf{g}$ is significantly smaller than the number of columns in $\mathbf{H}$, i.e. $m \ll n$, the direct inversion of the system is not feasible. Then, the compressed signal reconstruction is performed iteratively by finding a sparse solution to Eq. (2) given by the optimization problem expressed as

$\widehat{\boldsymbol{\theta}}=\underset{\boldsymbol{\theta}}{\operatorname{argmin}}\|\mathbf{g}-\mathbf{H} \boldsymbol{\Psi} \boldsymbol{\theta}\|_{2}^{2}+\tau\|\boldsymbol{\theta}\|_{1}$,

where $\|\cdot\| 2^{\wedge} 2$ is the square 1_2 -norm that measures the mean square error of the estimation, $\|\cdot\| \_1$ is the $1 \_1$-norm that measures the nonzero values in the vector and $\tau$ is a regulator which penalizes searching the sparsest solution. However, notice that the optimization problem presented in Eq. (3) does not consider the motion in the compressive measurements. In other words, this optimization problem searches a sparse solution for static images, hence, some motion artifacts are produced in the spectral video reconstruction process damaging the entire data and producing low spatial quality reconstruction.

\section{MULTIRESOLUTION RECONSTRUCTION APPROACH FOR COMPRESSIVE SPECTRAL VIDEO SENSING}

Briefly, the proposed multiresolution reconstruction approach is based on the reconstruction of a low spatial resolution version of the spectral video in order to extract the temporal or motion information. Then, the motion information is added as an additional regularization term in the optimization problem to correct motion artifacts and enhance the reconstruction quality of the high resolution spectral video.

More formally, for the low-resolution estimation, a spatial down-sampling operator $\mathbf{B} \in \mathbb{R}^{n / \kappa \times n},(n / \kappa \leq$ $n$ ), is introduced in Eq. (1) such that the measurements are rewritten as

$$
\mathbf{g}=\mathbf{H B}^{T} \mathbf{B f}
$$

where $\mathbf{B}^{T}$ is the transpose of $\mathbf{B}$. Then, an $\ell_{2}-\ell_{1}$-norm algorithm to solve the minimization problem presented in Eq. (3) is used with few iterations to obtain the sparsest coefficients of a coarse reconstruction of the spectral video from the measurements as

$$
\widehat{\boldsymbol{\theta}}_{\text {Low }}=\underset{\boldsymbol{\theta}}{\operatorname{argmin}}\left\|\mathbf{g}-\left(\mathbf{H B}^{T}\right) \mathbf{B}(\mathbf{\Psi} \boldsymbol{\theta})\right\|_{2}^{2}+\tau\|\boldsymbol{\theta}\|_{1} .
$$

Then, the low-resolution version of the spectral video is estimated by

$$
\hat{\mathbf{f}}_{\text {Low }}=\left(\boldsymbol{\Psi} \hat{\boldsymbol{\theta}}_{\text {Low }}\right)
$$

where $\quad \hat{\mathbf{f}}_{\text {Low }}=\left[\left(\hat{\mathbf{f}}_{0}^{T}\right)_{\text {Low }},\left(\hat{\mathbf{f}}_{1}^{T}\right)_{\text {Low }}, \ldots,\left(\mathbf{f}_{D-1}^{T}\right)_{L o w}\right]^{T}$ represents the low spatial resolution version.

The obtained coarse estimation $\hat{\mathbf{f}}_{\text {Low }}$ is up-sampled and then, used to extract the motion of the video as the optical flow.

The optical flow estimation between any two frames $\left(\hat{\mathbf{f}}_{w}\right)_{L o w}$ and $\left(\hat{\mathbf{f}}_{z}\right)_{L o w}$, for $w, z=0, \cdots D-1$ and $w>$ $z$, is given by $\overline{\mathbf{f}}_{w}=\mathbf{U}\left(\hat{\mathbf{f}}_{w}\right)_{\text {Low }}$, where $\mathbf{U}$ is an up-sampling operator such as a "bilinear interpolation". Then, the optical flow is estimated from any two frames $\overline{\mathbf{f}}_{w}$ and $\overline{\mathbf{f}}_{z}$ by computing the changes in the horizontal $u$ and vertical $v$ axis as in [14]. Then, for an estimation of the spectral video, the motion errors can be expressed as

$$
\boldsymbol{\Theta}=\Lambda\left(\overline{\mathbf{f}}_{w}\right)_{i, j}-\Lambda\left(\overline{\mathbf{f}}_{z}\right)_{(i+u),(j+v)},
$$

where $\Theta$ represents the error induced by the scene motion, $\Lambda(\cdot) \rightarrow\left\{\mathbb{R}^{N^{2}}: \rightarrow \mathbb{R}^{N \times N}\right\}$ is an operator that arranges a vector in matrix form, and $i, j$ goes over the spatial dimension of the selected frame.

Following the Eq. (7) to compute the motion errors, the optimization problem in Eq. (3) can be rewritten as

$$
\tilde{\boldsymbol{\theta}}=\underset{\boldsymbol{\theta}}{\operatorname{argmin}}\|\mathbf{g}-\mathbf{H} \boldsymbol{\Psi} \boldsymbol{\theta}\|_{2}^{2}+\tau\|\boldsymbol{\theta}\|_{1}+\beta\|\boldsymbol{\Gamma}\|_{2}^{2},
$$

where $\beta$ is a regularizer parameter and $\boldsymbol{\Gamma}$ is defined as

$$
\boldsymbol{\Gamma}=\Lambda(\boldsymbol{\Psi} \boldsymbol{\theta})_{i, j, k, w}-\Lambda(\boldsymbol{\Psi} \boldsymbol{\theta})_{(i+u),(j+v), k, z},
$$

where $\boldsymbol{\Psi} \boldsymbol{\theta}=\left[(\boldsymbol{\Psi} \boldsymbol{\theta})_{0}^{T}, \ldots,(\boldsymbol{\Psi} \boldsymbol{\theta})_{z}^{T},(\boldsymbol{\Psi} \boldsymbol{\theta})_{w}^{T}, \ldots(\boldsymbol{\Psi} \boldsymbol{\theta})_{D-1}^{T}\right]^{T}$ with $k=0, \ldots, L-1$. Notice that in Eq. (9) the subindex $(i+u)$ and $(j+v)$ accounts for the horizontal and vertical changes, respectively.

For illustration purposes, Figure 3 shows the optical flow estimation following a color map representation with the horizontal and vertical changes. Notice that in Fig. 3, the Frame 1 changes with respect to Frame 2 in the horizontal axis. Then, the optical flow representation adopts the respective colors given by the color map for the left and right movements [14].

To solve the problem in Eq. (8), it can be used wellknown implementations of signal recovery such as the LASSO or the GPSR algorithm by adding the 
regularization term as shown in Eq. (8) [15]. Finally, the reconstruction of the signal is attained by

$$
\tilde{\mathbf{f}}=\boldsymbol{\Psi} \tilde{\boldsymbol{\theta}},
$$

where $\tilde{\mathbf{f}}$ is the reconstructed spectral video in vector form.

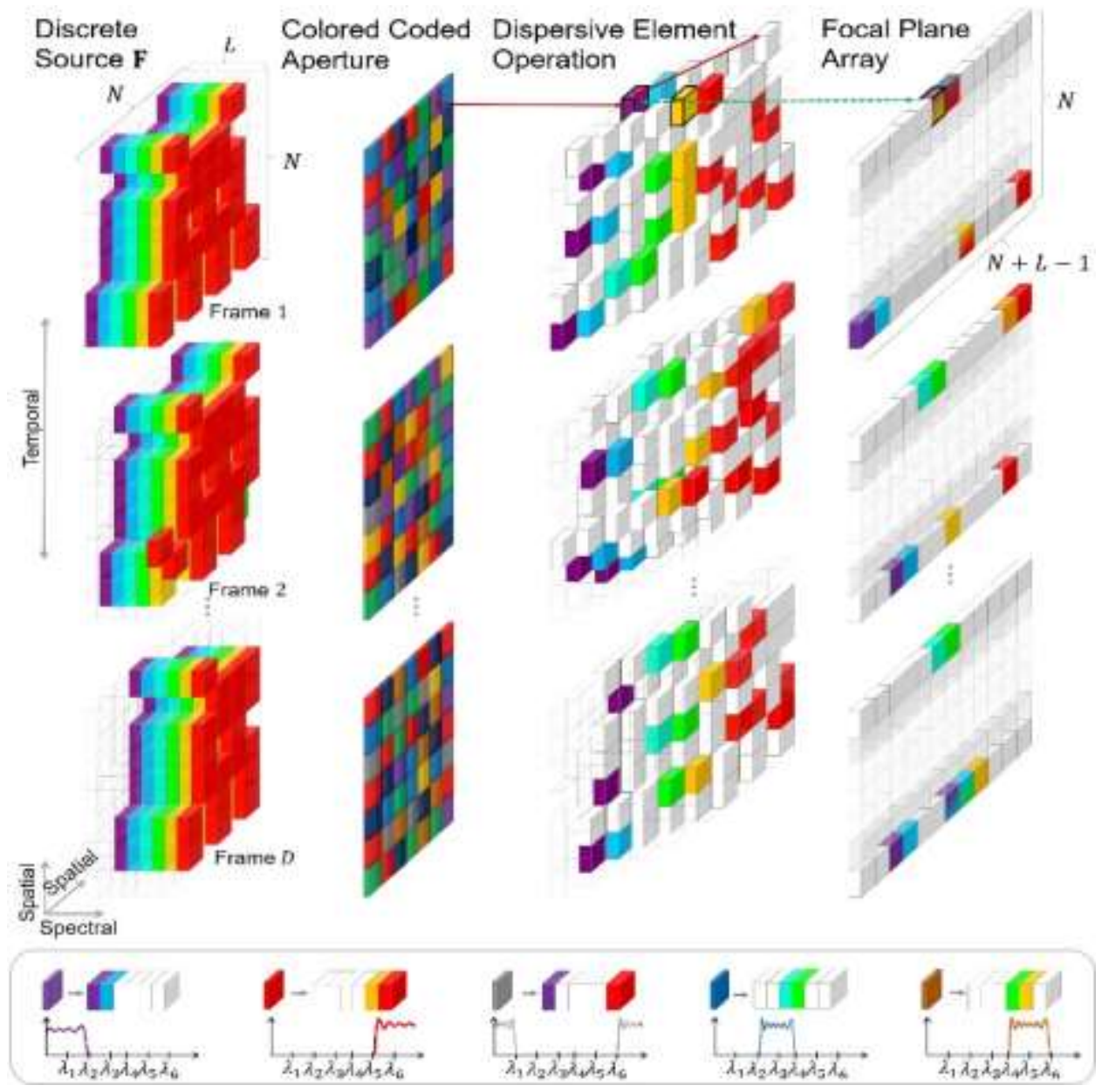

Figure 2. Illustration of the process in the video colored coded aperture-based compressive spectral imager (video C-CASSI). A set of optical filters used in the CCA is shown in the bottom.

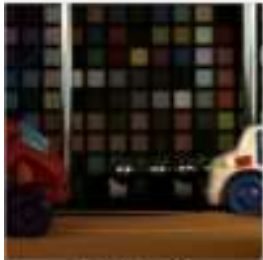

Frame 1

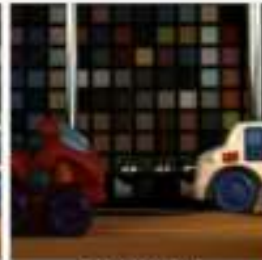

Frame 2

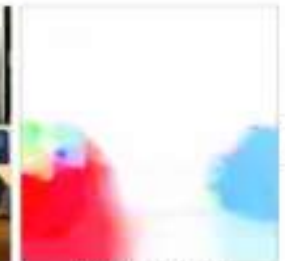

Optical flow representation

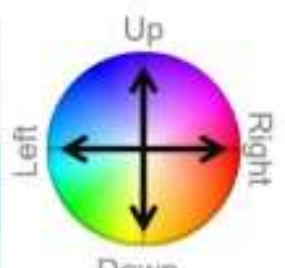

Down

Color Map

Figure 3. Illustration of the optical flow estimation between two frames following a color map. The scene changes from the Frame 1 to the Frame 2 and the optical flow representation adopts the respective color of the movement from the color map. 


\section{RESULTS}

To evaluate the performance of the proposed multiresolution reconstruction, a set of compressive measurements is simulated using the forward model in Eq. (1). For this, four test spectral videos were selected as follows. The first and the second dataset are cropped sections of the spectral video taken from [16] called Boxes 1 and Boxes 2, respectively. The third dataset, called Beads, is a synthetic spectral video of a moving object over a spectral static scene [17], and the fourth dataset, called Chiva bus, is a real sequence of spectral images acquired in the Optics Lab of the High Dimensional Signal Processing (HDSP) research group of the Universidad Industrial de Santander. All the datasets were acquired with a CCD camera and a VariSpec Liquid Crystal Tunable Filter (LCTF) in wavelengths from $400 \mathrm{~nm}$ to $700 \mathrm{~nm}$ at $10 \mathrm{~nm}$ steps. A spatial section of $N \times N$ pixels with $N=128, L=8$ spectral bands and $D=8$ frames was used for simulations. Specifically, the $L$ selected wavelengths were $\lambda_{0}=400, \lambda_{1}=440, \lambda_{2}=480, \lambda_{3}=520$, $\lambda_{4}=560, \lambda_{5}=600, \lambda_{6}=640$ and $\lambda_{7}=680 \mathrm{~nm}$. Figure 4 presents an RGB profile of the four test spectral videos.
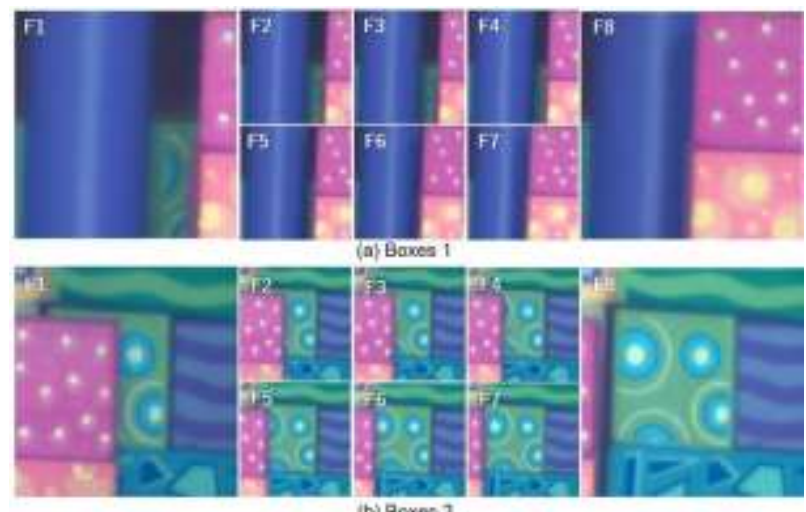
(b) Boxes 2

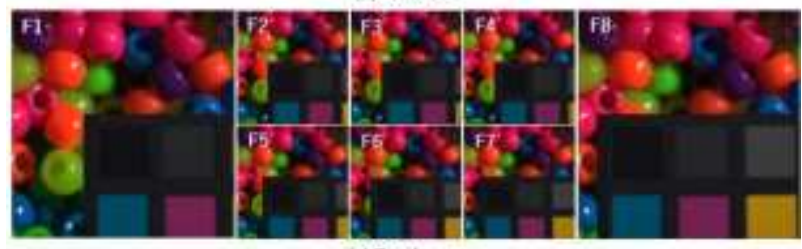

(c) Beacts

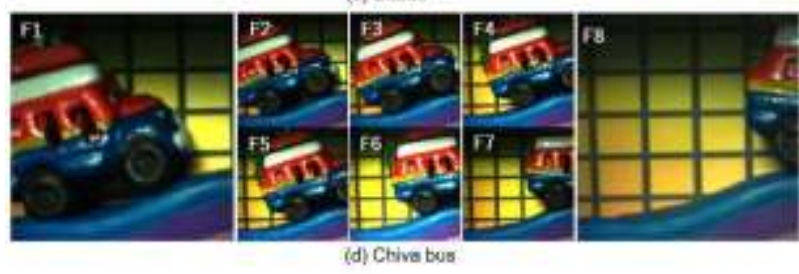

Figure 4. RGB representation of the eight frames for the four spectral videos used in the simulations. The resolution of each video is $128 \times 128$ spatial pixels, 8 spectral bands and 8 spectral frames.
For the numerical simulation of the video C-CASSI system illustrated in Fig. 1, it was used a random and an optimized colored coded aperture (CCA) with low and high band pass filters denoted by $\mathcal{L H}$-random CCA and $\mathcal{L H}$-CCA, respectively [1]. Further, in order to test the proposed multiresolution-based reconstruction, the different measurements attained with the aforementioned coded apertures realizations were reconstructed by using the GPSR algorithm adding the above-mentioned regularization term [15]. The peak signal-to-noise ratio (PSNR) metric is used to assess the image quality of the reconstructions. The PSNR is related with the mean square error (MSE) error as $10 \log _{10}\left(M A X^{2} / M S E\right)$ where $M A X$ is the maximum possible value of the image and the measure is given in decibels $(\mathrm{dB})$. All simulations were performed using the MATLAB software R2015a under the license Total Academic Headcount of the Universidad Industrial de Santander in an Intel Core i7 $3.6 \mathrm{GHz}$ processor and $16 \mathrm{~GB}$ RAM memory.

\subsection{Multiresolution reconstructions}

The low-resolution version for the multiresolution reconstruction was estimated with a spatial resolution of $32 \times 32$ pixels, i.e. a spatial down-sampling by a factor of 4 . The high-resolution reconstruction is performed following both the traditional and the proposed reconstruction by using the sparse solution attained from Eq. (3) and Eq. (8), respectively. Figure 5 shows an RGB representation of the frame 2 from the reconstructed videos (a) Boxes 1, (b) Boxes 2, (c) Beads and (d) Chiva bus with the proposed multiresolution reconstruction. For each coded aperture used, the averaged PSNR is shown.

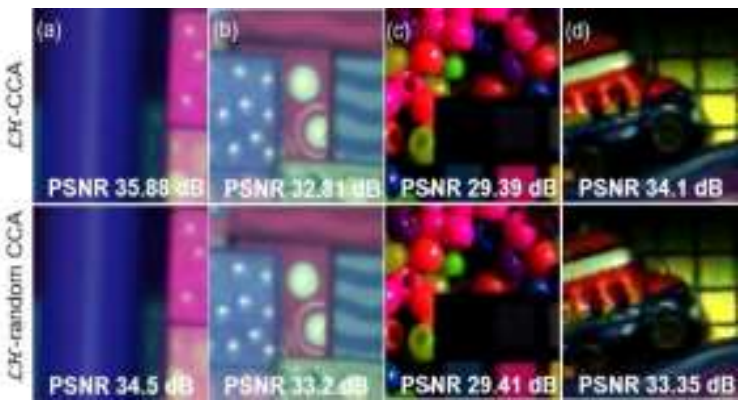

Figure 5. RGB representation of the frame 2 from the reconstructed videos: (a) Boxes 1, (b) Boxes 2, (c) Beads and (d) Chiva bus, using the proposed reconstruction approach. First row presents the reconstruction using $\mathcal{L} \mathcal{H}$-CCA, and second row using the $\mathcal{L} \mathcal{H}$-random CCA. The averaged PSNR across the spectral bands is shown for each case.

Figure 6 illustrates three original spectral bands for the 2nd frame of the Chiva bus video, and its respective reconstructions using the multiresolution approach. Each 
wavelength and the quality of reconstruction in terms of PSNR are shown.
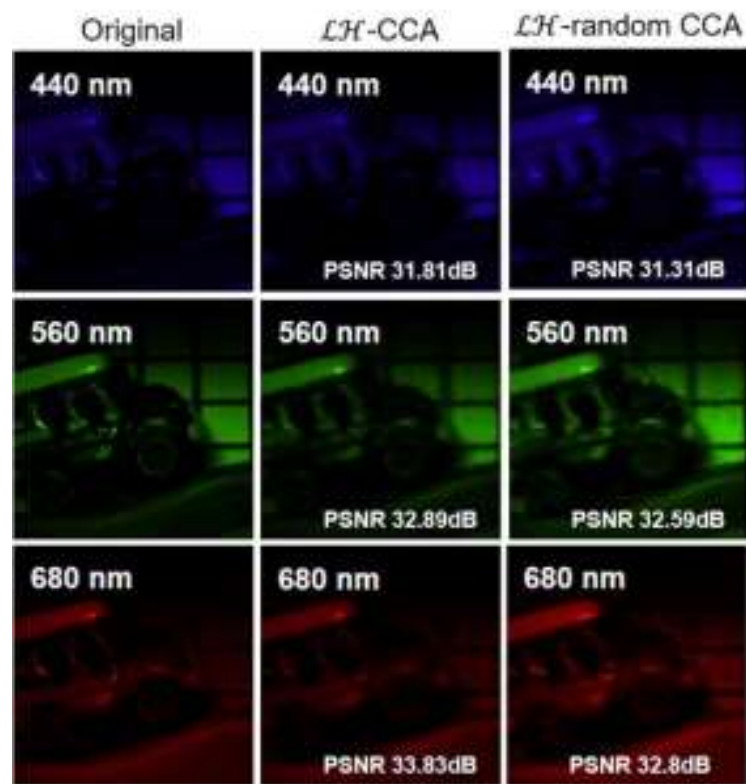

$680 \mathrm{~nm}$

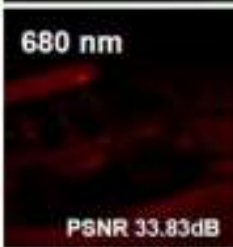

Figure 6. Original (first column) and reconstructions of 3 spectral bands for the frame 2 of the Chiva bus video by using $\mathcal{L} \mathcal{H}$-CCA (second column) and the $\mathcal{L} \mathcal{H}$-random CCA (third column) coded apertures. The quality reconstruction in terms of PSNR is shown for each spectral band.

\subsection{Multiresolution reconstruction vs. Traditional reconstruction}

Table 1 presents the summarization of the results in terms of averaged PSNR for the 2 coded aperture patterns used and both reconstruction methods. In general, observe that the obtained PSNR values with the multiresolution-based reconstruction are higher than those obtained with the traditional reconstruction.

Table 1. PSNR mean across the four dimensions for the traditional and multiresolution reconstruction.

\begin{tabular}{|c|c|c|c|c|}
\hline \multicolumn{5}{|c|}{ PSNR(dB) mean } \\
\hline $\begin{array}{l}\text { Traditional } \\
\text { reconstruction }\end{array}$ & Boxes 1 & Boxes 2 & Beads & $\begin{array}{c}\text { Chiva } \\
\text { bus }\end{array}$ \\
\hline$\overline{\mathcal{L H}-\mathrm{CCA}}$ & 32,17 & 31,6 & 26,68 & 30,07 \\
\hline$\overline{\mathcal{L H}}$-Random CCA & 31,46 & 31,6 & 26,41 & 30,2 \\
\hline $\begin{array}{l}\text { Multiresolution } \\
\text { reconstruction }\end{array}$ & & & & \\
\hline$\overline{\mathcal{L H}-\mathrm{CCA}}$ & 34,52 & $\mathbf{3 3 , 4 5}$ & $\mathbf{3 0 , 5 3}$ & 31,17 \\
\hline $\mathcal{L \mathcal { H }}$-Random CCA & 34,7 & 33,58 & 30,09 & 31,06 \\
\hline
\end{tabular}

Notice that the multiresolution-based reconstruction proposed outperforms the traditional reconstruction in up to $4 \mathrm{~dB}$.

\section{CONCLUSION}

The multiresolution reconstruction for compressive spectral video sensing in the video C-CASSI architecture has been proposed in this paper. For this, a low-resolution version is reconstructed using a few iterations of a $\ell_{2}-$ $\ell_{1}$-norm recovery algorithm. Later, from the lowresolution reconstruction, the optical flow is estimated to obtain the scene motion. The multiresolution-based reconstruction attempts to reduce the error originated by the temporal variable adding the scene motion as an additional regularization term in the minimization problem. Simulations show a reconstruction quality improvement using the proposed reconstruction up to 4 $\mathrm{dB}$ of PSNR with respect to the traditionally reconstruction.

\section{ACKNOWLEDGMENT}

The authors gratefully acknowledge the Vicerrectoría de Investigación y Extensión of Universidad Industrial de Santander for supporting this research registered under the project titled: "Diseño y simulación de un sistema adaptativo de sensado compresivo de secuencias de video espectral" (VIE code 1891).

\section{REFERENCIAS}

[1]H. Arguello and G. R. Arce, "Colored coded aperture design by concentration of measure in compressive spectral imaging," IEEE Transactions on Image Processing, vol. 23, no. 4, pp. 1896-1908, 2014.

[2]H. Rueda, H. Arguello, and G. R. Arce, "Dmd-based implementation of patterned optical filter arrays for compressive spectral imaging," JOSA A, vol. 32, no. 1, pp. 80-89, 2015.

[3]K. Leon, L. Galvis, and H. Arguello, "Reconstruction of multispectral light field (5d plenoptic function) based on compressive sensing with colored coded apertures from 2d projections," Revista Facultad de Ingeniería Universidad de Antioquia, no. 80, Ene. 2016.

[4]A. Wagadarikar, N. P. Pitsianis, X. Sun, and D. J. Brady, "Video rate spectral imaging using a coded aperture snapshot spectral imager," Optics Express, vol. 17, no. 8, pp. 6368-6388, 2009.

[5]C. Correa, D. F. Galvis, and H. Arguello, "Sparse representations of dynamic scenes for compressive spectral video sensing," Dyna, vol. 83, no. 195, p. 42, 2016.

[6]K. Leon, L. Galvis, and H. Arguello, "Spectral dynamic scenes reconstruction based in compressive sensing using optical color filters," in SPIE 
Commercial+ Scientific Sensing and Imaging. International Society for Optics and Photonics, 2016, pp. 98600D-98600D.

[7]B. Pedraza, P. Rondon, H. Arguello, "Sistema de reconocimiento facial basado en imágenes con color," Rev. UIS Ing., vol. 10, no. 2, 2012.

[8]A. B. Ramirez, H. Arguello, and G. Arce, "Video anomaly recovery from compressed spectral imaging," in Acoustics, Speech and Signal Processing (ICASSP), 2011 IEEE International Conference on. IEEE, 2011, pp. 1321-1324.

[9]J. Chen, Y. Wang, and H. Wu, "A coded aperture compressive imaging array and its visual detection and tracking algorithms for surveillance systems," Sensors, vol. 12, no. 11, pp. 14397-14415, 2012.

[10] A. Banerjee, P. Burlina, and J. Broadwater, "Hyperspectral video for illumination-invariant tracking," in Hyperspectral Image and Signal Processing: Evolution in Remote Sensing, 2009. WHISPERS'09. First Workshop on. IEEE, 2009, pp. 14.

[11] T. Goldstein, L. Xu, K. F. Kelly, and R. Baraniuk, "The stone transform: Multi-resolution image enhancement and compressive video," IEEE Transactions on Image Processing, vol. 24, no. 12, pp. 5581-5593, 2015.

[12] A. C. Sankaranarayanan, L. Xu, C. Studer, Y. Li, K. F. Kelly, and R. G. Baraniuk, "Video compressive sensing for spatial multiplexing cameras using motionflow models," SIAM Journal on Imaging Sciences, vol. 8, no. 3, pp. 1489-1518, Jul., 2015.

[13] D. Reddy, A. Veeraraghavan, and R. Chellappa, "P2c2: Programmable pixel compressive camera for high speed imaging," in Computer Vision and Pattern Recognition (CVPR), 2011 IEEE Conference on. IEEE, 2011, pp. 329-336.

[14] C. Liu, "Beyond pixels: exploring new representations and applications for motion analysis," Ph.D. dissertation, Citeseer, 2009.

[15] M. A. T. Figueiredo, R. D. Nowak, and S. J. Wright, "Gradient projection for sparse reconstruction: Application to compressed sensing and other inverse problems," IEEE Journal on Selected Topics in Signal Processing, vol. 1, no. 4, pp. 586-597, 2007.

[16] A. Mian and R. Hartley, "Hyperspectral video restoration using optical flow and sparse coding," Optics express, vol. 20, no. 10, pp. 10658-10673, 2012.
[17] F. Yasuma, T. Mitsunaga, D. Iso, and S. K. Nayar. 2008 CAVE Projects: Multispectral Image Database. [Online],Available:http://www.cs.columbia.edu/CAVE/ databases/multispectral/ 